\title{
A Technique to Improve MMSE Performance of PACE in Time-Selective Rayleigh Fading Channels
}

\author{
Rauf Iqbal, Thushara Abhayapala and Parastoo Sadeghi \\ Research School of Information Sciences and Engineering \\ Australian National University \\ Canberra, Australia \\ Emails: Rauf.Iqbal@anu.edu.au;Thushara.Abhayapala@anu.edu.au;Parastoo.Sadeghi@anu.edu.au
}

\begin{abstract}
The so-called peaky signaling has been shown to improve the performance of a communication system over noncoherent fading channels in the low signal-to-noise ratio (SNR) regime. We consider a signaling scheme which is equivalent to peaky signaling over a time-selective Rayleigh fading channel and make use of the unused degrees of freedom to improve the minimum mean square error (MMSE) performance of pilot-aided channel estimation (PACE) by a processing technique incorporating low pass filtering and downsampling. We assume infinite pilot symbols and optimal Wiener smoother at the receiver and give some analytical as well numerical results suggesting significant gains in MMSE performance of PACE.
\end{abstract}

\section{INTRODUCTION}

The use of pilot-aided channel estimation (PACE) in conjunction with non-peaky signaling is highly inefficient in the low signal-to-noise ratio (SNR) regime as half of the available power in a transmission block needs to be dedicated to pilot symbols [1, 2]. The use of pilot symbols with peaky signaling was shown to result in much improved performance in the low SNR regime [3] where channel was assumed to stay constant over a block of symbols. The peaky signaling concentrates most of the power in a very small number of degrees of freedom and remains silent for most of the time so that a large number of degrees of freedom are lost. It is of some interest to somehow make use of these unused degrees of freedom.

In this paper, we consider a time-selective
Rayleigh fading channel [1] where the channel dynamics are assumed to be characterized by ideal Ushaped Clarke's spectrum [4]. We consider a modified peaky signaling scheme with $M-1$ unused degrees of freedom of the original peaky signaling rearranged as shown in Fig. 1 where the factor $M$ can be considered as upsampling factor [5]. It is assumed that pilot symbols are inserted in the transmitted stream at least at the Nyquist rate i.e., $2 f_{\mathrm{D}}$ where $f_{\mathrm{D}}$ is the channel fading rate normalized to the symbol rate, $1 / T_{s}$. At the receiver, the received symbol stream is processed as shown in Fig. 2 to obtain minimum mean square error (MMSE) estimate of channel gain at data symbols.

The rest of the paper is organized as follows. In Section II we describe the discrete time Rayleigh fading model along with different parameters which will be the basis of the subsequent analysis. In Section III we give transfer function for the optimal Wiener filter and corresponding MMSE. The results of Section III are used in Section IV to numerically evaluate the gains offered by the proposed scheme. Finally, the conclusion is drawn in Section V.

\section{Channel Model}

The channel between the transmitter and the mobile receiver is modeled as a stationary frequency non-selective circularly-symmetric complex Gaussian random process. The output $r[m]$ of the matched filter at symbol time $m$ in complex base- 


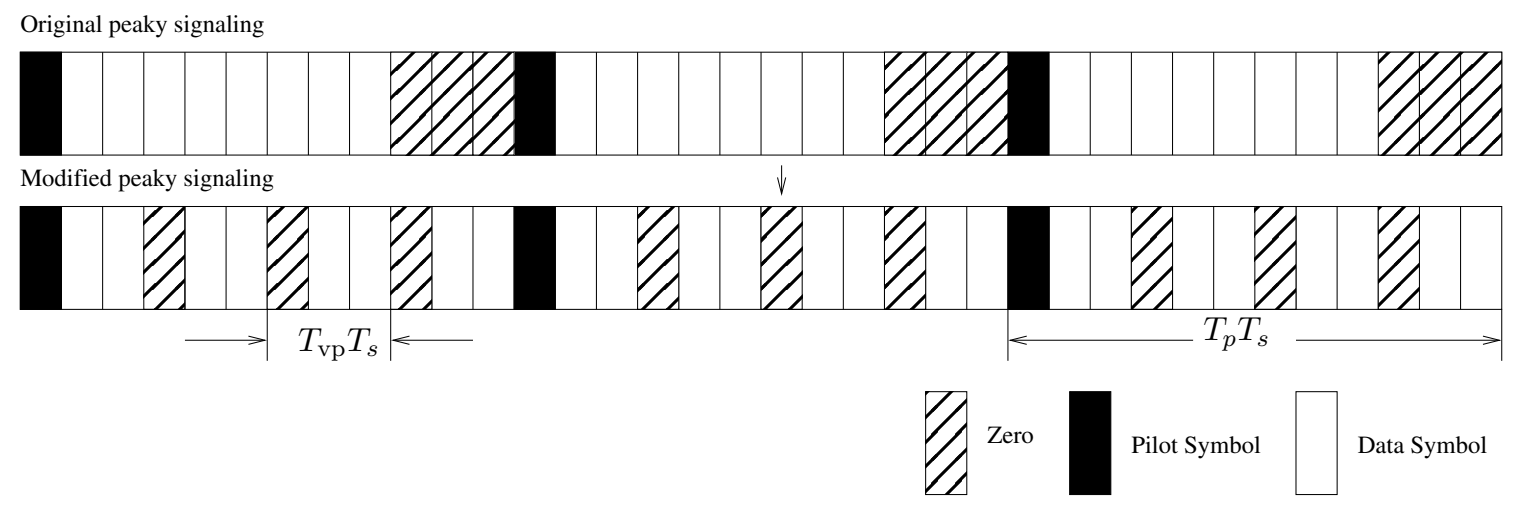

Fig. 1. Transmitted frame format $\left(T_{s}\right.$ is the data symbol duration and $T_{p}$ denotes pilot symbol insertion factor. The number of unused degrees of freedom is equal to $M-1$.

band equivalent form can be written as

$$
r[m]=\sqrt{\mathcal{E}_{s}} h[m] s[m]+z[m],
$$

where $s[m], h[m]$ and $z[m]$ (assumed to be independent) are the samples of complex channel input symbol, channel gain and additive discrete white Gaussian noise (AWGN) at signaling interval $m$. Equation (1) is normalized such that $\mathcal{E}\left\{|s[m]|^{2}\right\}=$ $1, \mathcal{E}\left\{|h[m]|^{2}\right\}=1$ and $\mathcal{E}\left\{|z[m]|^{2}\right\}=N_{0}$, and $\mathcal{E}_{s} / N_{0}$ represents the average received signal-tonoise ratio (SNR) per data symbol. The channel statistics are assumed to be known to the receiver.

We assume that the symbol rate is such that

$$
\frac{1}{T_{\mathrm{p}}}=K 2 f_{\mathrm{D}}
$$

where integer $K \geq 1$ to ensure at least Nyquist rate sampling of the channel. We also assume that the cut-off frequency, $f_{c}$ of the low pass filter in Fig. 2 is given as

$$
f_{c}=f_{\mathrm{D}} \frac{T_{p}}{M}
$$

If $x[n]$ is the output during pilot symbol at $n$th pilot symbol instant, we have

$$
x[n]=r\left[n T_{p}\right]=\sqrt{\mathcal{E}_{p}} h\left[n T_{p}\right]+z\left[n T_{p}\right],
$$

where $\mathcal{E}_{p}$ is the average power per pilot symbol. Since we wish to obtain MMSE estimate at data symbol locations which are shifted with respect to pilot symbol locations, we need to define the following shifted and sampled channel process

$$
c_{k}[n]=h\left[n T_{p}+k\right], \quad 0 \leq k \leq T_{p}-1
$$

where $c_{k}[\cdot]$ represents the channel gain at data symbol at delay of $k$ symbols from the pilot symbol at instant $n T_{\mathrm{p}}$.

\section{Design of Optimal Wiener Smoother AND CORRESPONDING MMSE}

In order to explore the ultimate performance of the proposed scheme, we input noisy pilot sequence $x[n]$ to the Wiener filter (Fig. 2) which is optimal in the mean-square sense. We know from classical Wiener filter theory [6] that the transfer function of optimal infinite impulse response (IIR) MMSE Wiener filter $W_{o, k}$ is given in frequency domain as

$$
W_{o, k}(\omega)=\frac{\mathcal{S}_{c_{k} x}(\omega)}{\mathcal{S}_{x x}(\omega)},
$$

where $\mathcal{S}_{c_{k} x}(\omega)$ is the cross-spectral density of the pilot observation process and channel gain at data symbol shifted $k$ symbols from the pilot symbol, and $\mathcal{S}_{x x}(\omega)$ is the spectral density of the pilot observation process. Following the approach of [7], we can show that the optimal Wiener filter and the corresponding MMSE, $\sigma_{\epsilon}^{2}$ for the proposed scheme 


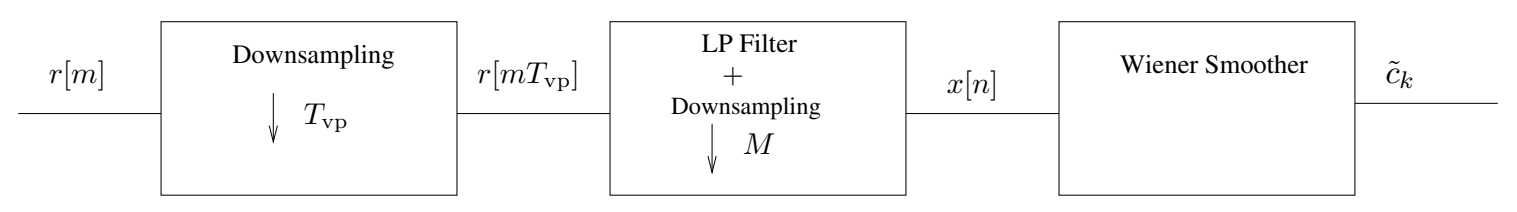

Fig. 2. Proposed scheme of channel estimation using pilot symbols.

are given respectively as

$$
\begin{gathered}
W_{o, k}(\omega)=e^{j \omega k} \frac{1}{T_{p}} \frac{\sqrt{\mathcal{E}_{p}} \mathcal{S}_{h}\left(\frac{\omega}{T_{p}}\right)}{\frac{N_{0}}{T_{p}} \mathcal{S}_{h}\left(\frac{\omega}{T_{p}}\right)}, \\
\sigma_{\epsilon}^{2}= \\
\int_{-2 \pi f_{\mathrm{D}}}^{2 \pi f_{\mathrm{D}}} \frac{\mathcal{S}_{h}(\omega)}{2 \pi}\left(1-\frac{\mathcal{E}_{p} \mathcal{S}_{h}(\omega)}{T_{p}\left(\frac{N_{0}}{M}+\frac{\mathcal{E}_{p}}{T_{p}} \mathcal{S}_{h}(\omega)\right)}\right) d \omega,
\end{gathered}
$$

where the factor $e^{j \omega k}$ in (6) is due to the crossspectral density, $\mathcal{S}_{c_{k} x}(\omega)$. Notice the presence of additional (downsampling) factor $M$ in (6) and (7).

To keep the analysis simple, we consider the uniform Doppler spectrum, $\mathcal{S}_{h h}^{U}(\omega)$, which results when the mobile receiver is surrounded by isotropic 3D scattering [8] and classical U-shaped Clarke's spectrum, $\mathcal{S}_{h h}^{C}(\omega)$, which results when there is isotropic scattering in 2D [4]. The respective MMSE for the uniform and Clarke's Doppler spectra can be shown to be given as

$$
\begin{gathered}
\sigma_{U, \epsilon}^{2}=\frac{2 f_{\mathrm{D}} T_{p}}{2 f_{\mathrm{D}} T_{p}+M \mathbb{S N R}_{p}} \\
\sigma_{C, \epsilon}^{2}=1-\frac{4 \alpha}{\pi \sqrt{1-\alpha^{2}}} \operatorname{arctanh}^{-1}\left(\sqrt{\frac{1-\alpha}{1+\alpha}}\right),
\end{gathered}
$$

where $\alpha:=M \mathbb{S N R}_{p} /\left(\pi f_{\mathrm{D}} T_{p}\right)$.

In order to draw a comparison of the MMSE performance of the proposed scheme and other schemes, we define the following:

$$
\begin{aligned}
\mathbb{M M S E}_{g} & =\sigma_{\mathrm{NF}}^{2}-\sigma_{\mathrm{F}}^{2}, \\
& \geq 0,
\end{aligned}
$$

where we have used $\sigma_{\mathrm{NF}}^{2}$ and $\sigma_{\mathrm{F}}^{2}$ respectively to indicate MMSE for schemes without filtering and the proposed scheme. For a fixed $M$ and $f_{\mathrm{D}}$, it can be shown that $\mathbb{M M S E}_{g}$ for a uniform channel doppler spectrum, $\mathbb{M M S E}_{g}^{U}$ is maximum when

$$
\mathbb{S N R}_{p}^{\mathrm{U} \max }=\frac{2 f_{\mathrm{D}} T_{p}}{\sqrt{M}}
$$

which implies that when $f_{\mathrm{D}}$ is small, the proposed scheme offers the most at lower $\mathbb{S N R}_{p}$.

Notice that $\mathrm{b}$ y discarding $M-1$ degrees of freedom, we are able to save power meant for discarded degrees of freedom. While the foregoing analysis is applicable in all possible scenarios, due to paper length restrictions, we shall concentrate in the following section on the scenario where the power savings are used solely to enhance pilot symbol power (i.e., pilot enhancement (PE) case).

\section{Numerical ANALYSis of $\mathbb{M M S E}_{g}$}

The behavior of $\mathbb{M M S E}_{g}$ function against $\mathbb{S N R}_{p}$ for different values of $M$ has been plotted in Fig. 3 for uniform Doppler spectrum. We can observe significant improvement in MMSE performance for $M=10$ and 20 especially at low $\mathbb{S N R}_{p}$. With pilot enhancement (assuming data symbol power of just $1 \mathrm{~dB}$ ) and sufficiently large $M$, MMSE performance improvement is quite significant for very low $\mathbb{S N R}_{p}$. Notice that the peakiness of the signaling scheme (which is proportional to $M$ ) can result in better MMSE even at moderate $\mathbb{S N R}_{p}$ implying that for $\mathbb{S N R}_{p}<\infty$, it is always advantageous from the point of view of MMSE performance to lose some degrees of freedom. However, for pilot-based systems, better MMSE performance does not imply a gain in overall information transmission rate.

The impact of normalized fading rate on MMSE performance for a fixed $\mathbb{S N R}_{p}=-10 \mathrm{~dB}$ has been shown in Fig. 4 for different values of $M$ for the case of PE. The depicted behavior of 


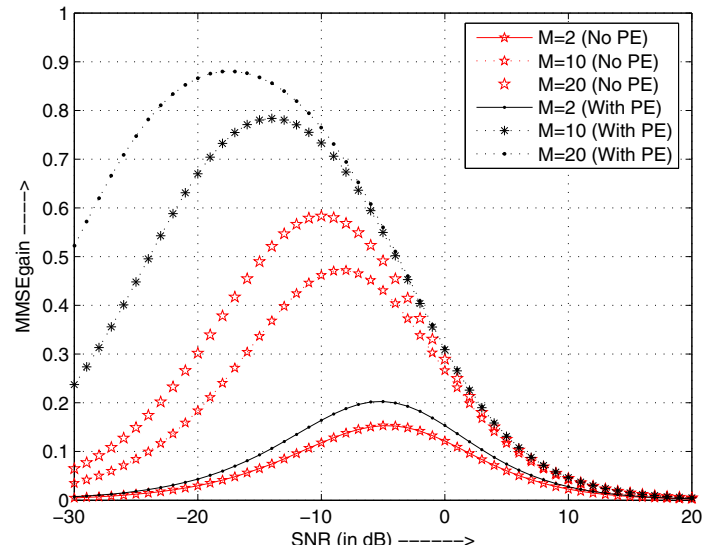

Fig. 3. Impact of SNR on $\mathbb{M M S E}_{g}$ with and without PE when $f_{\mathrm{D}}=0.005$ and $K=2$ for Clarke's spectra. The power per data symbol is assumed to be $1 \mathrm{~dB}$ (normalized to 1 unit of power).

MMSE performance can be explained in view of the fact that we have assumed lowpass filtering of noisy pilot symbols such that the channel spectrum is unaltered which implies that the filtering (and downsampling) operation does not alter power of the channel process. However, the noise power is altered in inverse proportion to $f_{\mathrm{D}}$. In other words, a change in $f_{\mathrm{D}}$ does not change the (useful) signal power but changes the noise power thus changing $\mathbb{S N R}_{p}$. Therefore, a change in $f_{\mathrm{D}}$ is equivalent to changing $\mathbb{S N R}_{p}$ and the impact of change in $\mathbb{S N R}_{p}$ is plotted in Fig. 3 for Clarke's spectrum.

\section{CONClusion And Future Work}

We considered modified peaky signaling over continuously fading Rayleigh channel. We proposed low pass filtering followed by downsampling of pilot symbol stream at the receiver making use of the unused degrees of freedom in the peaky signaling. The added processing boosted pilot symbol SNR which translated into improved MMSE performance of the channel estimation scheme. It turned out that MMSE gain offered by the proposed scheme depends on SNR, fading rate, pilot insertion frequency and peakiness of the signaling scheme. While this contribution has focussed on MMSE gain

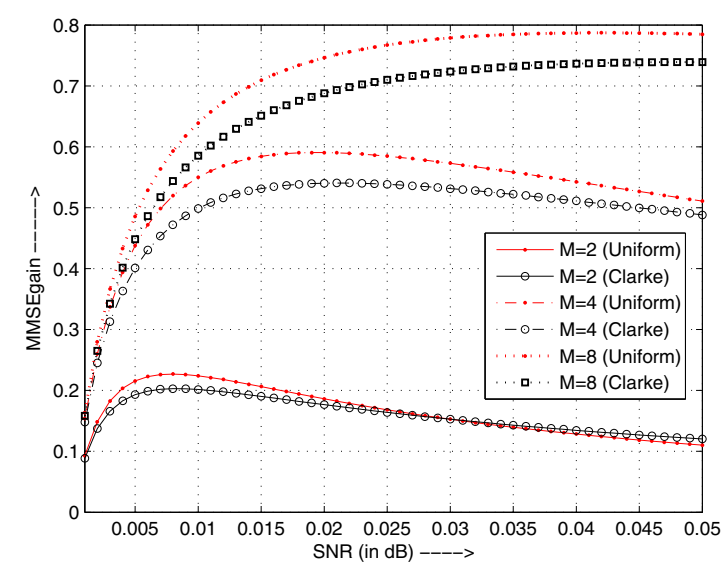

Fig. 4. Impact of normalized fading rate, $f_{\mathrm{D}}$ on $\mathbb{M M S E}_{g}$ with $\mathrm{PE}$ for different $M$ when $\mathrm{SNR}_{p}=-10 \mathrm{~dB}$ for uniform and Clarke's spectra. The power per data symbol is assumed to be 1 $\mathrm{dB}$ and $T_{p}=10$.

due to the proposed scheme, it will be of some interest to see how this improved MMSE performance translates into gains in real-world communication system resources like bandwidth and power when we seek to improve overall system performance.

\section{REFERENCES}

[1] X. Deng and A. M. Haimovich, "On pilot symbol aided channel estimation for time varying Rayleigh fading channels," in Proceedings of Conference on Information Sciences and Systems (CISS04), pp. 78-82, March 2004.

[2] B. Hassibi and B. M. Hochwald, "How much training is needed in multiple-antenna wireless links?" vol. 49, no. 4, pp. 951-963, Apr. 2003.

[3] L. Zheng, M. Médard, D. Tse, and C. Luo, "Channel coherence in the low SNR regime," in International Symposium on Information Theory (ISIT). IEEE, June-July 2004, 2007, pp. 976-997.

[4] R. H. Clarke, "A statistical theory of mobile-radio reception," Bell Syst. Tech. J., vol. 47, no. 6, pp. 957-1000, 1968.

[5] A. V. Oppenheim and R. Shafer, Eds., Discrete-Time Signal Processing, 2nd ed. New Jersey: Prentice Hall, 1989.

[6] T. Kailath, A. Sayed, and B. Hassibi, Linear Estimation. Prentice-Hall, 2000.

[7] K. Yu, J. Evans, and I. Collings, "Performance Analysis of Pilot Symbol Aided QAM for Rayleigh Fading Channels," in IEEE International Conference on Communications, 2002, pp. 1731-1735.

[8] R. H. Clarke and W. L. Khoo, "3-(D) mobile radio channel statistics," in IEEE Transactions on vehicular technology, vol. 46, no. 3, 1997, pp. 798-799. 